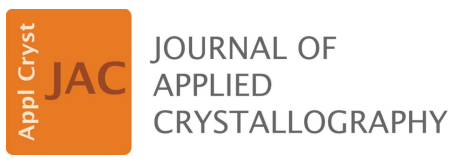

ISSN 1600-5767

Keywords: editorial; ptychography; imaging.

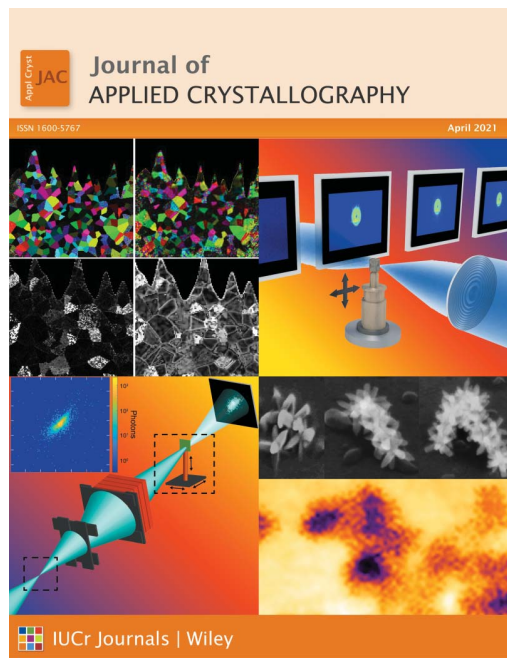

\section{Introduction to the special issue on Ptychography: software and technical developments}

\author{
Stefano Marchesini, ${ }^{a}$ David Shapiro ${ }^{\mathrm{b}}$ and Filipe R. N. C. Maia ${ }^{\mathrm{c}, \mathrm{d} *}$ \\ ${ }^{a}$ Computational Research Division, Lawrence Berkeley National Laboratory, Berkeley, CA 94720, USA, b Advanced Light \\ Source, Lawrence Berkeley National Laboratory, Berkeley, CA 94720, USA, 'Department of Cell and Molecular Biology, \\ Uppsala University, 75124 Uppsala, Sweden, and ${ }^{\text {d} N E R S C, ~ L a w r e n c e ~ B e r k e l e y ~ N a t i o n a l ~ L a b o r a t o r y, ~ B e r k e l e y, ~ C A ~}$ \\ 94720, USA. *Correspondence e-mail: filipe@xray.bmc.uu.se
}

Ptychography is an X-ray technique that achieves resolutions of one billionth of a metre, can reach a macroscopic field of view, and has the capability to retrieve chemical or magnetic contrast, among other features. It includes variants such as ptycho-tomography and spectro-ptychography, which are some of the most computationally intensive techniques being developed at X-ray light sources around the world.

In the past few years ptychography has grown into a diverse landscape of methods to address ever more complex systems. These techniques involve beautiful mathematics, from harmonic analysis and algebraic geometry to complex numerical analysis.

The Ptycho Developers 2019 workshop held at Berkeley Laboratory on 3-5 June 2019 was attended by 60 participants from five continents. The goal of the workshop was to bring together developers and practitioners of ptychography to assess the current landscape of available algorithms and software, investigate commonalities, and discuss a range of topics, from required theoretical and algorithmic advancements and unmet needs to practical issues of implementation and deployment. Other topics included workflows, beamlines, optics and high-performance computing solutions.

Several ideas presented at the Berkeley workshop were later turned into manuscripts. These manuscripts formed the seed that grew into this virtual special issue, being complemented by diverse contributions from around the world.

This special issue provides us with an opportunity to take a glimpse of some of the developments addressed in the workshop. The 14 articles it contains cover a broad variety of subjects but can be roughly divided into the following groups:

(1) A new speckle-based ptychography variant is introduced by Morgan, Murray, Prasciolu et al. (2020), aimed at wavefront metrology. This is accompanied by two further manuscripts, headed by the same author: one of them (Morgan, Murray, Quiney et al., 2020) describing speckle-tracking, an open-source software package for the analysis of data from the newly developed X-ray speckle-tracking ptychography variant, and the other (Morgan, Quiney et al., 2020) showcasing its first application, aiding the fabrication of wedged multi-layer Laue lenses by providing a way to accurately characterize them.

(2) Du et al. (2020) address the dose requirements of multiple X-ray microscopy techniques with detailed numerical simulations and conclude that it is the photon fluence, and not the experimental geometry, that limits the resolution of a properly conducted measurement.

(3) Two manuscripts highlight a broad range of direct applications of ptychography. De Caro et al. (2020) use it to image some peculiarly shaped nanocrystals in a polymer matrix. Sala et al. (2020) show us how ptychography can be used with a range of sources and a variety of purposes by imaging the wavefront of an X-ray free-electron laser.

(4) Significant advances in instrumentation are presented by three manuscripts. Khant et al. (2020) show us that NanoMAX is ready for ptycho-tomographic measurements, taking advantage of the high coherent flux delivered by the diffraction-limited storage ring of MAX IV. Schropp et al. (2020) introduce us to PtyNAMi, which stands for ptychographic nano-analytical microscope, and chronicle how the P06 beamline at PETRA III has been converted into a state-of-the-art hard X-ray ptychography instrument. Pound et al. (2020) tell us how ptychography can be carried out parasitically at an $\mathrm{X}$-ray free-electron laser, demonstrating it at the Coherent X-ray Imaging endstation of the Linac Coherent Light Source. Beamtime at such facilities is even more constrained than at synchrotrons due to their serial nature, so the ability to carry out multiple experiments simultaneously will be very valuable. 
(5) Two manuscripts by Chang and co-workers (Chang, Marcus \& Marchesini, 2020; Chang, Rong et al., 2020) tackle the theory and algorithms for spectral ptychography and dichroic ptychography.

(6) Lastly, there were several contributions showcasing new computer codes and software, as was to be expected from a developers' conference. Baraldi et al. (2020) describe highly optimized software tools for fast ptychographic reconstructions, particularly suited to the high-speed fly scans that have been developed at Sirius. Wakonig et al. (2020) introduce PtychoShelves, a high-performance modular framework for ptychographic reconstructions developed at the Paul Scherrer Institute, but which has been tested at a variety of light sources including home sources and electron sources. Finally, FavreNicolin et al. (2020) announced an updated version of $P y N X$, a highly flexible operator-based toolkit for coherent X-ray imaging related tasks, from data simulation to analysis. The new version includes a complete overhaul of the ptychography-related code as well as increased use of GPUs for better performance.

There is no doubt that the Ptycho Developers 2019 workshop helped push forward the field and we hope to see future editions of the workshop as soon as we are able to gather together again. We very much look forward to more developments to broaden the scope and increase the speed, power and user-friendliness of ptychography.

The virtual special issue is available at https://journals. iucr.org/special_issues/2021/ptycho.

\section{References}

Baraldi, G. L., Dias, C. S. B., Silva, F. M. C., Tolentino, H. C. N. \& Miqueles, E. X. (2020). J. Appl. Cryst. 53, 1550-1558.
Chang, H., Marcus, M. A. \& Marchesini, S. (2020). J. Appl. Cryst. 53, 1283-1292.

Chang, H., Rong, Z., Enfedaque, P. \& Marchesini, S. (2020). J. Appl. Cryst. 53, 937-948.

De Caro, L., Scattarella, F., Altamura, D., Arciniegas, M. P., Siliqi, D., Manna, L. \& Giannini, C. (2020). J. Appl. Cryst. 53, 741-747.

Du, M., Gürsoy, D. \& Jacobsen, C. (2020). J. Appl. Cryst. 53, 748759.

Favre-Nicolin, V., Girard, G., Leake, S., Carnis, J., Chushkin, Y., Kieffer, J., Paleo, P. \& Richard, M.-I. (2020). J. Appl. Cryst. 53, 1404-1413.

Kahnt, M., Sala, S., Johansson, U., Björling, A., Jiang, Z., Kalbfleisch, S., Lenrick, F., Pikul, J. H. \& Thånell, K. (2020). J. Appl. Cryst. 53, 1444-1451.

Morgan, A. J., Murray, K. T., Prasciolu, M., Fleckenstein, H., Yefanov, O., Villanueva-Perez, P., Mariani, V., Domaracky, M., Kuhn, M., Aplin, S., Mohacsi, I., Messerschmidt, M., Stachnik, K., Du, Y., Burkhart, A., Meents, A., Nazaretski, E., Yan, H., Huang, X., Chu, Y. S., Chapman, H. N. \& Bajt, S. (2020). J. Appl. Cryst. 53, $927-$ 936.

Morgan, A. J., Murray, K. T., Quiney, H. M., Bajt, S. \& Chapman, H. N. (2020). J. Appl. Cryst. 53, 1603-1612.

Morgan, A. J., Quiney, H. M., Bajt, S. \& Chapman, H. N. (2020). J. Appl. Cryst. 53, 760-780.

Pound, B. A., Mertes, K. M., Carr, A. V., Seaberg, M. H., Hunter, M. S., Ward, W. C., Hunter, J. F., Sweeney, C. M., Sewell, C. M., Weisse-Bernstein, N. R., Baldwin, J. K. S. \& Sandberg, R. L. (2020). J. Appl. Cryst. 53, 1276-1282.

Sala, S., Daurer, B. J., Odstrcil, M., Capotondi, F., Pedersoli, E., Hantke, M. F., Manfredda, M., Loh, N. D., Thibault, P. \& Maia, F. R. N. C. (2020). J. Appl. Cryst. 53, 949-956.

Schropp, A., Döhrmann, R., Botta, S., Brückner, D., Kahnt, M., Lyubomirskiy, M., Ossig, C., Scholz, M., Seyrich, M., Stuckelberger, M. E., Wiljes, P., Wittwer, F., Garrevoet, J., Falkenberg, G., Fam, Y., Sheppard, T. L., Grunwaldt, J.-D. \& Schroer, C. G. (2020). J. Appl. Cryst. 53, 957-971.

Wakonig, K., Stadler, H.-C., Odstrčil, M., Tsai, E. H. R., Diaz, A., Holler, M., Usov, I., Raabe, J., Menzel, A. \& Guizar-Sicairos, M. (2020). J. Appl. Cryst. 53, 574-586. 